\title{
Mitro-Aortic Valve-in-Valve Procedures: Many Challenges in Little Space
}

\author{
Marco Gennari ${ }^{1}$, Piero Trabattoni ${ }^{1}$, Gianluca Polvani ${ }^{2}$, and Marco Agrifoglio ${ }^{1}$ \\ ${ }^{1}$ Centro Cardiologico Monzino Istituto di Ricovero e Cura a Carattere Scientifico \\ ${ }^{2}$ Affiliation not available
}

May 18, 2020

\begin{abstract}
Redo multiple valve replacement is known to carry additional risk of morbidity and mortality. Currently, a transcatheter-based valve-in-valve approach could be useful in reducing potential serious consequences. On the other hand, this approach poses several technical challenges regarding the device and procedural aspects of the procedure. We present the case of a 78-year-old man who presented with symptoms of heart failure due to mitro-aortic bioprosthesis degenerations who was deemed to be at extremely high risk for conventional redo surgery. A two-steps single admission transcatheter-based approach was planned with a transfemoral aortic valve-in-valve procedure followed by a trans-apical mitral valve-in-valve implantation. The outcome was good and the recovery was fast.
\end{abstract}

\section{Introduction}

Re-interventions for degenerated bioprosthetic valves are known to carry additional risk of postoperative morbidity and mortality; in elderly patients aged $>75$ years this is particularly evident with an estimated postoperative risk of mortality that may exceed $10 \%$. [1]

Transcatheter procedures have proven to be a safe alternative to conventional redo cardiac surgery in complex redo cases although they pose additional challenges especially in the setting of valve-in-valve deployment due to the close anatomical relationships of the mitro-aortic valves.

We report a successful two-steps valve-in-valve procedure for the replacement of failed mitro-aortic bioprosthesis.

\section{Case Presentation}

A 78-year- old male presented to our Emergency Department for two weeks history of shortness of breath and peripheral swelling. He had a previous medical history of several cardio-vascular risk factors, moderate renal insufficiency (last creatinine level $1.45 \mathrm{mg} / \mathrm{dL}$ ), polyarthritis of chronic steroids and permanent atrial fibrillation with near normal left atrial dimensions. In 2001 he had undergone mitro-aortic bioprostetic replacement for rheumatic disease with a $29 \mathrm{~mm}$ and $25 \mathrm{~mm}$ Magna Edwards bovine prosthesis (Edwards Lifesciences, Irvine, $C A$ ), respectively. The transthoracic echocardiogram showed a depressed left ventricular ejection fraction (LVEF) of $28 \%$ in the setting of severe mitro-aortic stenosis, moderate tricuspid insufficiency and preserved right ventricular function. The patient was deemed to be at unacceptable high risk for a conventional redo surgery due to a Euroscore II risk of $16.81 \%$ and a Society of Thoracic Surgeon (STS) score of $13.09 \%$ and an elevated frailty index. He underwent multi detector computed tomography (MDCT) in order evaluate the technical feasibility of the transcatheter approach (Figure 1 ). Coronary evaluation showed moderate left anterior descending stenosis (60\%). 
Finally, the Heart team approved a two-steps transcatheter approach. We chose a transfemoral percutaneous aortic valve-in-valve procedure with a $26 \mathrm{~mm}$ Sapien 3 valve (Edwards Lifesciences, Irvine, CA ) under local anesthesia and conscious sedation as first step. In the same admission, after ten days and computed tomography reassessment of the prosthesis relationships, we performed a mitral valve-in-valve procedure with a transapical approach (left antero-lateral minithoracotomy) with an off-label implantation of a reversed 29 mm Sapien 3 prosthesis (Edwards Lifesciences, Irvine, $C A$ ). (Figure 2 ). The postoperative course was uneventful, and the patient was quickly discharged home. He is alive and well at 2-year follow-up.

\section{Discussion}

Valve-in-valve $(\mathrm{ViV})$ procedures have become a viable alternative to treat high risk redo surgical patients either in the aortic and mitral position. There are sparse reports of staged or concomitant transfemoral or transfemoral and transeptal replacements. [2,3]

Several technical aspects must be considered. On the aortic side it is mandatory an accurate valve sizing to reduce the risk of patient-prosthesis mismatch (PPM) and to guarantee a maximal valvular sealing; for a balloon-expandable device it is generally recommended a $1 \mathrm{~mm}$ minimum oversizing respect the internal stent diameter of the failed bioprosthesis.

Regarding the mitral replacement, the key aspects for a successful implantation are the correct sizing (similar to the aortic one) and the correct height of implantation to reduce the risk of atrial valve migration or left ventricular outflow tract (LVOT) obstruction. On this regard, an acute mitro-aortic angle $<115$ degrees and a neo-LVOT area less than $250 \mathrm{~mm}^{2}$ are commonly considered as pre-procedural risk factors for LVOT obstruction. [4]

In this case we decided to perform the staged two-steps approach following these considerations.

We have firstly replaced the aortic bioprosthesis to relief the afterload, improve cardiac output and diastolic coronary perfusion (CT evidence of moderate proximal left anterior descending stenosis).

Secondly, the patient presented with a severely depressed LVEF thus we preferred to postpone the transapical approach to let some degree of ventricular improvement In fact, the first postoperative echocardiogram showed an increased left ventricular ejection fraction (EF 35\%). Due to the persistence of small left atrial dimensions although permanent atrial fibrillation we chose to not pursuit the transseptal approach and we planned a standard transapical route.

Finally, the delayed second procedure allowed us to re-assess the geometrical relationships between the mitro-aortic struts and choose the optimal site of transapical approach for better mitral co-axiality.

In conclusion, patients with previous history of mitro-aortic surgery can be very challenging in case of either redo surgery or transcatheter replacement, due to the biological prosthesis's relationships, although there is still a lack of a specific risk score [5] for this particular cohort of patients.

An in-depth geometrical preoperative and postoperative investigation has proven to be a useful and reproducible tool leading to optimal technical and at the end clinical outcomes. We strongly believe that in the structural heart interventions field a close patient-to-patient approach in a qualified heart team is the only way to perform high quality procedure with low grade of risk, in a high-level healthcare service.

\section{References}

1. M Maganti M, V Rao V, Armstrong S, Feindel CM, Scully HE and David TE. Redo Valvular Surgery in Elderly Patients. Ann Thorac Surg 2009;87:521-6.

2. Rene AG, Desai N, Anwaruddin S. Concomitant transfemoral transcatheter aortic and mitral valvein-valve replacement. J Card Surg. 2017;32:479-482.

3. Schlömicher M, Haldenwang PL, Moustafine V, Bechtel M, Strauch JT. Simultaneous double valve-invalve TAVI procedure for failed bioprostheses. Ann Thorac Surg. 2015;99(2):722-724. 
4. Shivaraju A, Michel J, Frangieh AH, Ott I, Thilo C, Schunkert H. et al. Transcatheter Aortic and Mitral Valve-in-Valve Implantation Using the Edwards Sapien 3 Heart Valve. J Am Heart Assoc. 2018;7:e007767.

5. Aziz M, Simonato M, Webb JG, et al. Mortality prediction after transcatheter treatment of failed bioprosthetic aortic valves utilizing various international scoring systems: Insights from the Valve-inValve International Data (VIVID). Catheter Cardiovasc Interv. 2018;92(6):1163-1170.

\section{Figures}

Figure 1. Preoperative computed tomography assessment. The close relationship between the two bioprosthetic struts and the mitro-aortic angle appear clear.

Figure 2. Fluoroscopic images of the aortic valve-in valve $(\mathbf{A}, \mathbf{B})$ and the subsequent mitral valve-in-valve procedure $(\mathbf{C})$.

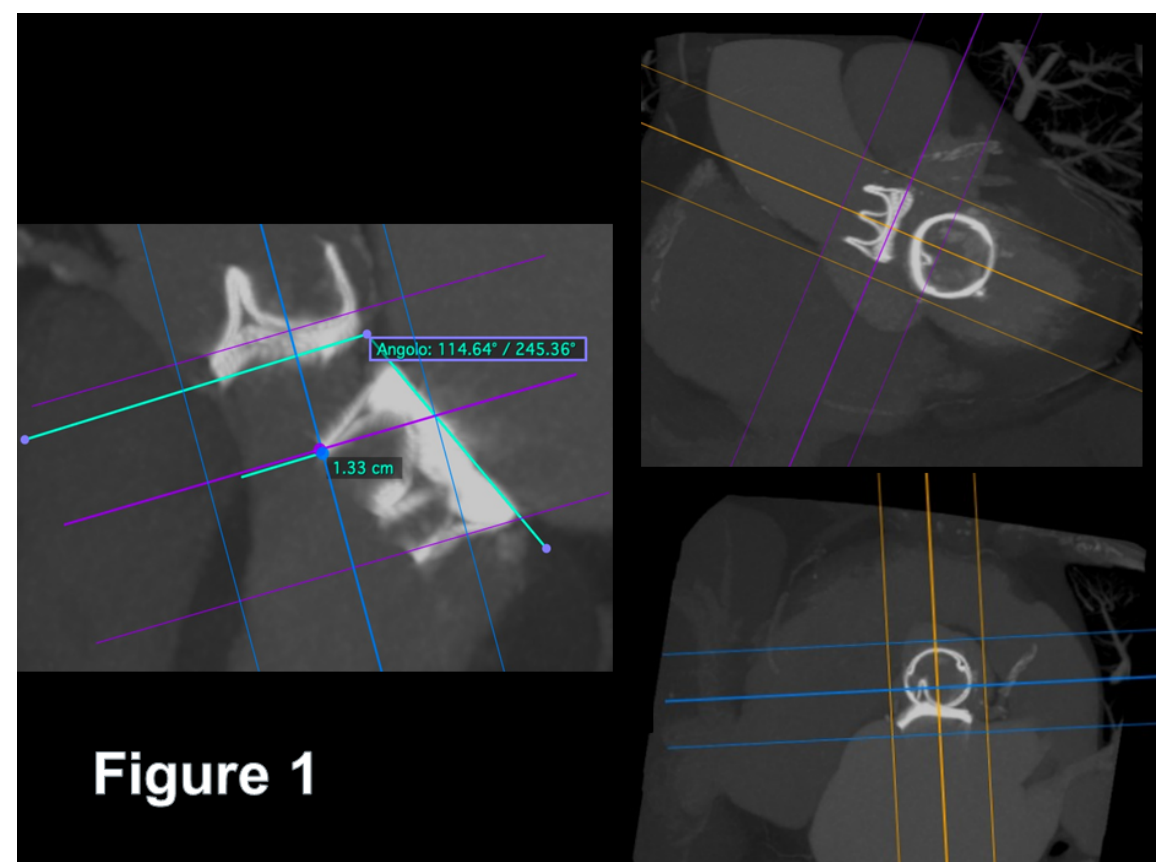




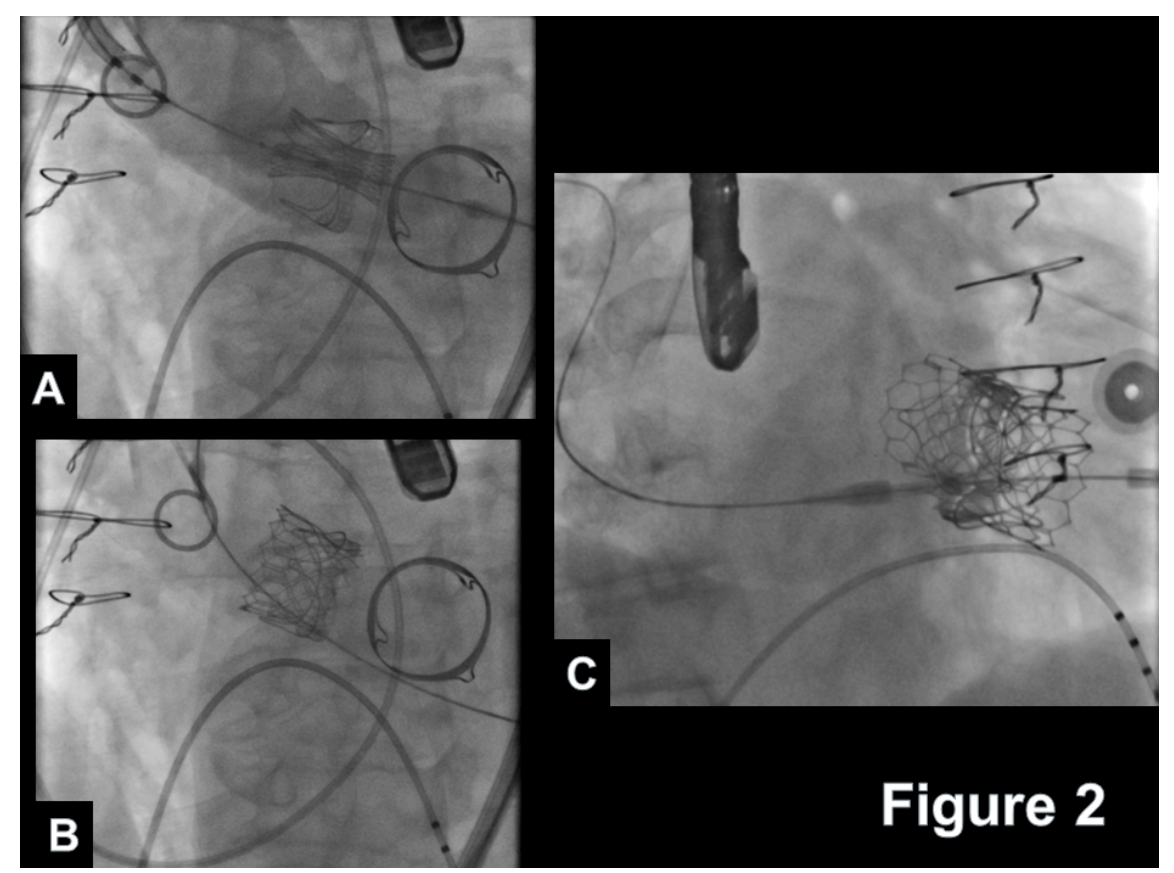

